\title{
Rapid diagnosis of beta-haemolytic streptococcus group B endocarditis by buffy-coat examination and gas-liquid chromatography
}

\author{
N. MALIWAN, R. REID, AND R. KATZEN \\ From A mbulatory Care Service and the Departments of Medicine and Pathology, the Veterans \\ Administration Medical Center, Hines, Illino is 60141 and the Abraham Lincoln School of Medicine, the \\ University of Illinois College of Medicine, Chicago, Illinois 60680, USA
}

SUMMARY A semicomatose 34-year-old man had fever, heart murmur, and multiple gangrenous lesions. Several blood cultures were negative. Cultures of the heart valve grew beta-haemolytic streptococcus group B. The organism was seen in buffy-coat examination of the venous blood. The gas-liquid chromatogram of the serum was different from those of normal controls and of patients with invasive candida infection; it closely mimicked the one obtained from the organism itself. These studies promise to be valuable diagnostic adjuncts.

The diagnosis of infective endocarditis is usually based on the clinical presentation of fever, heart murmur, splenomegaly, and thromboembolic phenomena together with positive blood cultures. Problems arise when some of the signs are not present and especially if blood cultures are negative, which occurred in $7-24 \%$ of cases in a review by Durack and Petersdorf (1977). This may be due to the fastidious nature of the organism or, more commonly, to the prior use of antibiotics. Non-infectious conditions such as myxoma or marantic endocarditis mimic infective endocarditis very closely. This and the wide range of causative organisms together with the side effects of many antimicrobial agents make the definitive identification of the organism extremely desirable.

\section{Case report}

A 34-year-old white man was admitted to Hines Veterans Administration Hospital on 12 April 1977 from another hospital. Approximately two weeks before admission he had been treated elsewhere with cephalexin and tetracycline for symptoms of an upper respiratory tract infection. On 9 April he was found unconscious on the bathroom floor and was taken to hospital. The findings at that time included 'frostbite' of both feet and bilateral basilar infiltrates. He responded only to painful stimuli. A sputum culture grew Staphylococcus epidermidis and yeasts.

Received for publication 8 January 1979
Two blood cultures showed no growth. The patient was initially placed on cephalothin and lincomycin; later, the treatment was changed to chloramphenicol. There was no improvement in his general condition and he was transferred to Hines VAH three days later.

On admission his temperature was $39.5^{\circ} \mathrm{C}$, heart rate $104 / \mathrm{min}, \mathrm{BP} 140 / 70 \mathrm{mmHg}$, and respiratory rate $28 / \mathrm{min}$. The patient was semicomatose and had a right hemiplegia and nuchal rigidity. The pupils were equal and reactive, and the fundi contained multiple haemorrhagic lesions without papilloedema. A petechial haemorrhage was noted on the left conjunctiva, and multiple petechiae were found over the anterior chest wall. Both feet, the fourth distal phalanx of the right hand, and the skin overlying the right knee were cold and showed dry gangrenous changes. The right dorsalis pedis pulse was absent and the left was barely palpable. Examination of the heart revealed an $\mathbf{S}_{4}$ and a grade II-III/VI systolic murmur at the apex. Auscultation of the lungs revealed bilateral rhonchi. The liver span was $12 \mathrm{~cm}$ and the spleen was not palpable. A chest $x$-ray showed a mild cardiomegaly and a possible right lower lobe infiltrate. Laboratory tests showed a mild leucocytosis with left shift, microscopic haematuria without casts, and a slight increase in liver enzymes.

\section{HOSPITAL COURSE}

The patient was started on cephalothin and gentamicin with a provisional diagnosis of bacterial endocarditis. Spinal tap showed clear xanthochromic 
fluid with an opening pressure of $12 \mathrm{~cm}$, protein 79 $\mathrm{mg} / \mathrm{dl}$, glucose $41 \mathrm{mg} / \mathrm{dl}$, WBC 6/mm ${ }^{3}$ (all lymphocytes), RBC $2 / \mathrm{mm}^{3}$; no organisms were seen on a Gram stain. Cultures of spinal fluid and three blood specimens showed no growth. Examination of freshly obtained sputum showed many budding and mycelial forms of fungi, later identified on culture as Candida species. A skin biopsy of the right knee lesion was negative for bacteria and fungi by Gram and PAS stains. An echocardiogram and right heart catheterisation were compatible with vegetative endocarditis involving the mitral valve. On the third day in hospital he underwent excision of the mitral valve together with a Björk Shiley valve replacement. Preoperatively amphotericin $B$ was added to the drug regimen. This was discontinued postoperatively after the staining of the excised valve showed only Gram-positive cocci in pairs and short chains. These were identified culturally as beta-haemolytic streptococcus group B. The patient was then placed on heparin, penicillin, and gentamicin. The fever started to come down but the rest of the vital signs remained unchanged until the third postoperative day when he developed atrial fibrillation. Two days later he had a respiratory arrest after which time he had to bemaintained on a respirator and intravenous dopamine drip. The pupils became fixed and dilated and serial EEGs $\times 3$ showed no cortical activity. He was pronounced dead 11 days after admission.

A post-mortem examination revealed multiple infarcts of the spleen, kidneys, and lungs and massive intracerebral and intraventricular haemorrhages with diffuse softening of the brain.

\section{SPECIAL INVESTIGATION}

Two special studies were made on the venous blood samples obtained from the first day of admission to Hines VAH:

1 buffy-coat examination: a Wright-Giemsa stain of the buffy coat revealed large numbers of monocytes; some of them contained coccoid bodies intracellularly, ranging in numbers from one to six per cell (Fig. 1);

2 gas-liquid chromatography (GLC).

A Beckman gas chromatograph no. 45 equipped with a flame ionisation detector was used. The glass column, $2 \mathrm{~mm}$ in internal diameter by $1.8 \mathrm{~m}$ in length, was packed with $3 \%$ methyl silicone gum rubber (SE-30) on Gas-Chem Q, 100-120 mesh (Applied Science Laboratories, Inc, State College, PA 16801, USA). Carrier gas was helium and was operated at $40 \mathrm{ml} / \mathrm{min}$. The temperature at the injection port was $235^{\circ} \mathrm{C}$ and $230^{\circ} \mathrm{C}$ for the detector. For the latter, hydrogen and air flow was at the rate of $38 \mathrm{ml} / \mathrm{min}$ and $300 \mathrm{ml} / \mathrm{min}$ respectively. For separation and detection, the column was maintained isothermally at $170^{\circ} \mathrm{C}$ until the peaks had been resolved. Lypopure methanol, hexamethyldisilazane, trimethylchlorosilane, and lypopure pyridine were obtained from Applied Science Laboratories, Inc.

Streptococcus group B whole cells were prepared by using an overnight culture in a yeast nitrogen

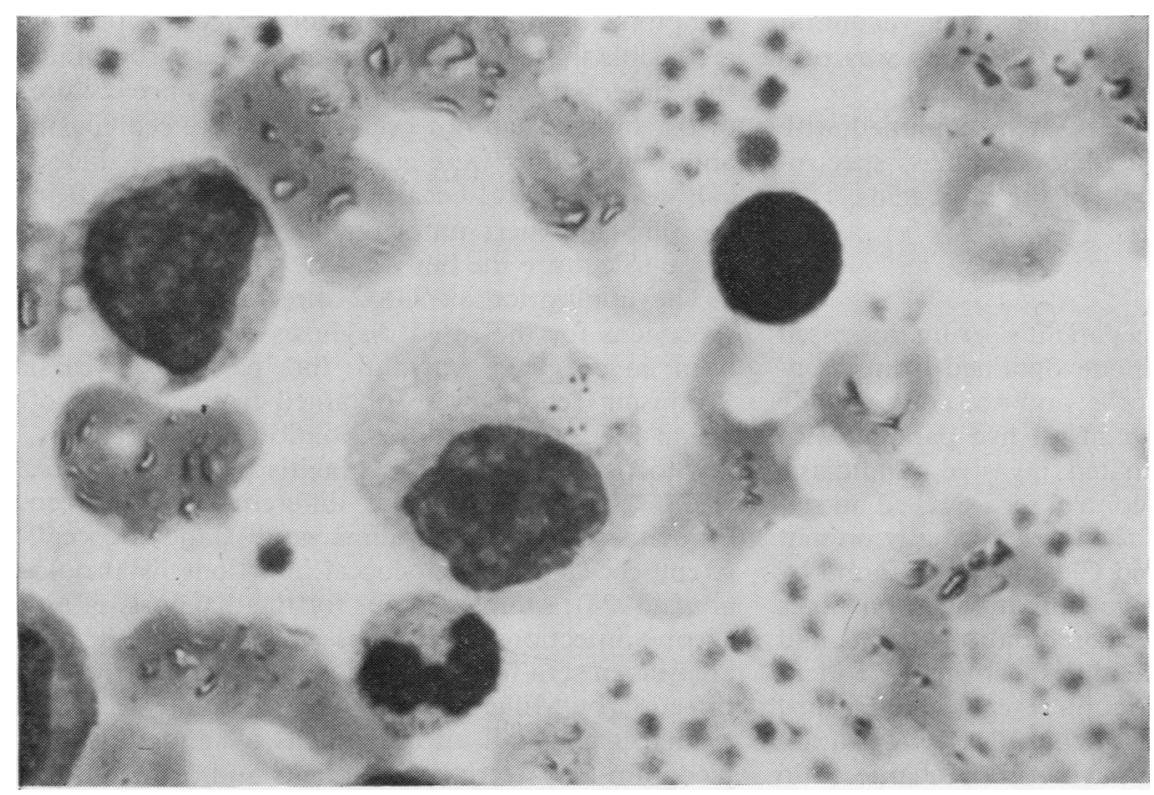

Fig. 1 Wright-Giemsa stain of the buffy coat shows six coccoid bodies within a large monocyte, shown in the centre of the picture. 


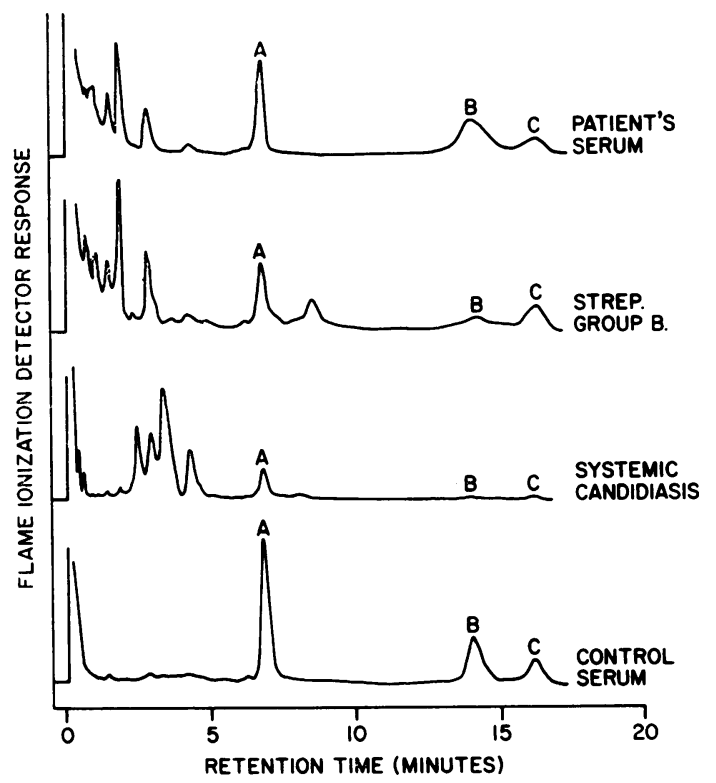

Fig. 2 Gas-liquid chromatograms of the patient's serum, streptococcus group B cells suspension, and one sample each from patients with systemic candidiasis and normal controls. Note the distinctive pattern of the patient's serum, which closely resembles that of streptococcus group B cells and is easily distinguishable from other conditions.

broth centrifuged at $10000 \mathrm{~g}$. The sediments were washed three times in normal saline and dried overnight over silica gel in a desiccator under vacuum.

Trimethylsilyl derivatisation of samples was performed according to Miller et al. (1974).

A Finnigan $4000 \mathrm{GC} / \mathrm{MS}$ system equipped with chemical ionisation was used for the mass spectrometric confirmation of some of the fatty acids.

\section{Result}

The chromatogram of this patient's serum possessed a striking similarity to the one obtained from streptococcus group B whole cells, and was contrastingly different from the pattern of all five patients with histology-proven disseminated invasive candidiasis (Fig. 2). All nine normal control sera showed no significant peaks in front of the three normally present peaks designated $\mathrm{A}, \mathrm{B}$, and $\mathrm{C}$, which were found by co-chromatography to have similar retention times to those of palmitic acid, a combination of oleic and linoleic acids and stearic acid, respectively. The identity of the fatty acids in the control sera was confirmed by GC/MS analyses using methane as the ionisation gas. They were shown to have a mass charge ratio
(M/e) of 271, 298, and 300, which corresponded to the $M+1$ ions of the methyl esters of the aforementioned fatty acids.

\section{Discussion}

In this case the previous use of multiple antibiotics coupled with signs of large-vessel embolisations initially suggested the possibility of candida endocarditis. Scanty reports on the demonstration of candida in the buffy coat, ie, by Silverman et al. (1973), and the rapid identification of candida sepsis by GLC by Miller et al. (1974) prompted our study. The findings from both were quite rewarding although the organism did not prove to be candida as originally suspected.

A recent report by Lerner et al. (1977) on group B streptococcus infections, which included a review of the literature, disclosed 13 cases of endocarditis from 1938 to 1945 and 15 cases since 1962 . All the preantibiotic cases occurred acutely in women during the postpartum or postabortal period. The mitral valve was involved in 12 cases and the aortic valve in one; large vegetations were noted in $8 / 9$ necropsies, and embolisation to the major vessels was common. Among cases found since 1962, a subacute form with more frequent involvement of the aortic valve and one case of tricuspid valve involvement were noted; also both sexes were equally affected.

Underlying conditions were found to include recent birth, pre-existing valvular disease, diabetes mellitus, cellulitis, and respiratory or genitourinary tract infection. Our patient initially presented with symptoms of an upper respiratory tract infection. It is possible that he acquired streptococcus group B at that time, but this could not be proved since culture of the sputum had not been done before cephalexin and tetracycline were given. The negative blood cultures on several occasions could be explained in the same manner; unfortunately, no attempt was made to culture the buffy coat.

The application of GLC directly on biological specimens for the rapid diagnosis of infections has been investigated only in the past few years. Promising reports were obtained from analysis of spinal fluids for the differentiation of tuberculous, cryptococcal, and viral meningitis by Craven et al. (1977); synovial fluids for differentiation between synovitis caused by trauma and staphylococcal, streptococcal, and gonococcal infections by Brooks et al. (1974); samples of pus for the diagnosis of anaerobic infections by Phillips et al. (1976); and the analysis of sera for the diagnosis of invasive candida infection by Miller et al. (1974). The ability of GLC to detect as small an amount as a nanogram and picogram of bacterial constituents and/or metabolic 
products makes it a probable tool for diagnosing even localised infections. Rapid analysis directly from sera, if applicable, has great advantage since appropriate treatment can be rendered without the usual need for potentially harmful invasive procedures to obtain specimens from anatomical sites of varying accessibility. Miller et al. (1974), however, could not differentiate bacteraemic sera from normal controls, as previously reported by Mitruka et al. (1972). The heavy load of organisms in bacterial endocarditis as in our case is possibly responsible for our positive findings. The rapidity of the tests and lack of interference from the prior use of antibiotics reinforce the value of buffy-coat examination and especially of GLC in the diagnosis of bacterial endocarditis with negative blood cultures. Additional advantages of GLC include its sensitivity and specificity. Using serum specimens, we have produced promising results from cases of tuberculosis and staphylococcal and disseminated invasive candida sepsis (in preparation).

\section{References}

Brooks, J. B., Kellogg, D. S., Alley, C. C., Short, H. B , Handsfield, H. H., and Huff, B. (1974). Gas chromatography as a potential means of diagnosing arthritis. I. Differentiation between staphylococcal, streptococcal, gonococcal, and traumatic arthritis. Journal of Infectious Diseases, 129, 660-668.

Craven, R. B., Brooks, J. B., Edman, D. C., Converse, J. D., Greenlee, J., Schlossberg, D., Furlow, T.,
Gwaltney, J. M., Jr., and Miner, W. F. (1977). Rapid diagnosis of lymphocytic meningitis by frequencypulsed electron capture gas-liquid chromatography: differentiation of tuberculous, cryptococcal, and viral meningitis. Journal of Clinical Microbiology, 6, 27-32.

Durack, D. T., and Petersdorf, R. G. (1977). Changes in the epidemiology of endocarditis. In Infective Endocarditis. An American Heart Association Symposium, edited by E. L. Kaplan and A. V. Taranta, pp. 3-8. American Heart Association, Dallas, Texas.

Lerner, P. I., Gopalakrishna, K. V., Wolinsky, E., McHenry, M. C., Tan, J. S., and Rosenthal, M. (1977). Group B. streptococcus ( $S$. agalactiae) bacteremia in adults: analysis of 32 cases and review of the literature. Medicine, 56, 457-473.

Miller, G. G., Witwer, M. W., Braude, A. I., and Davis, C. E. (1974). Rapid identification of Candida albicans septicemia in man by gas-liquid chromatography. Journal of Clinical Investigation, 54, 1235-1240.

Mitruka, B. M., Kundargi, R. S., and Jonas, A. M. (1972). Gas chromatography for rapid differentiation of bacterial infections in man. Medical Research Engineering, 11 (2), 7-11.

Phillips, K. D., Tearle, P. V., and Willis, A. T. (1976). Rapid diagnosis of anaerobic infections by gas-liquid chromatography of clinical material. Journal of Clinical Pathology, 29, 428-432.

Silverman, E. M., Norman, L. E., Goldman, R. T., and Simmons, J. (1973). Diagnosis of systemic candidiasis in smears of venous blood stained with Wright's stain. American Journal of Clinical Pathology, 60, 473-475.

Requests for reprints to: Dr N. Maliwan, Hines VA Medical Center, PO Box 1017, Hines, Illinois 60141, USA. 